\title{
POLYNOMIAL APPROXIMATION TO HARMONIC AND ANALYTIC FUNCTIONS: GENERALIZED CONTINUITY CONDITIONS
}

\author{
BY \\ J. L. WALSH AND H. MARGARET ELLIOTT(')
}

1. Introduction. Class $\Lambda^{*}$. In the study of approximation to a function $F(\theta)$ of the real variable $\theta$ by trigonometric polynomials $T_{n}(\theta)$ of order $n$, Lipschitz conditions of order $\alpha(0<\alpha<1)$ play a central role, for a necessary and sufficient condition that $F(\theta)$ (assumed to have the period $2 \pi$ ) can be uniformly approximated on the interval $0 \leqq \theta \leqq 2 \pi$ by trigonometric polynomials $T_{n}(\theta)$ with the degree of approximation $1 / n^{\alpha}$ is that $F(\theta)$ satisfy a Lipschitz condition of order $\alpha$. This proposition is false in the case $\alpha=1$, and no requirement on a modulus of continuity is necessary and sufficient in that case. Recently Zygmund [1]( $\left.{ }^{2}\right)$ has replaced the Lipschitz condition of order 1 by the condition

$$
|F(\theta+\delta)+F(\theta-\delta)-2 F(\theta)| \leqq M|\delta|,
$$

where $M$ is independent ${ }^{(3)}$ of $\theta$ and $\delta$; the class $\Lambda^{*}$ is the class of all continuous functions $F(\theta)$ of period $2 \pi$ which satisfy (1.1). Zygmund proves an elegant and important result:

THEOREM 1.1. A necessary and sufficient condition that a function $F(\theta)$ with period $2 \pi$ can be uniformly approximated by trigonometric polynomials $T_{n}(\theta)$ with the degree of approximation $1 / n$ is that $F(\theta)$ belong to $\Lambda^{*}$.

Likewise in the theory of approximation to an analytic function $f(z)$ by polynomials $\pi_{n}(z)$ of degree $n$ in the complex variable $z$, Lipschitz conditions continue to play a central role; if $C$ is an analytic Jordan curve, a necessary and sufficient condition that $f(z)$ analytic interior to $C$ and continuous in the corresponding closed region $\bar{C}$ can be uniformly approximated in $\bar{C}$ by polynomials $\pi_{n}(z)$ with the degree of approximation $1 / n^{\alpha}(0<\alpha<1)$ is that $f(z)$ satisfy in $\bar{C}$ a Lipschitz condition of order $\alpha$. Again the case $\alpha=1$ is known to be exceptional. It is the primary purpose of the present paper to treat this case $\alpha=1$, and to show that the analogue of condition (1.1) provides a necessary and sufficient condition that $f(z)$ can be uniformly approximated in $\bar{C}$ by polynomials $\pi_{n}(z)$ with the degree of approximation $1 / n$. Closely related to

Presented to the Society, September 2, 1949; received by the editors September 1, 1949.

(1) This paper was written while Dr. Elliott was a Vassie James Hill A.A.U.W. Fellow.

(2) References in brackets refer to the bibliography at the end of the paper.

( $\left.{ }^{3}\right)$ The letters $K, L$, and $M$ with or without subscripts will always denote constants which may vary from one inequality to another and which may depend on the point sets involved, but which are always independent of $w, z, \zeta, \theta, s, \partial, s, \delta$, and $h$. In addition the constants $L$ and $M$ are always independent of $m$ and $n$. 
approximation to analytic functions is approximation to harmonic $\left({ }^{4}\right)$ functions by harmonic polynomials; we show that here too condition (1.1) is of similar importance. The two topics of approximation, to analytic functions by polynomials in the complex variable and to harmonic functions by harmonic polynomials, are unified and integrated in a recent paper [Walsh, Sewell, and Elliott 1]; the methods there developed with suitable modification are the chief methods on approximation used in the present paper.

The writers are indebted to Professor Zygmund for having drawn their attention to the possible application of his results to the problem of approximation in the complex domain.

We introduce

DeFinItion 1.1. Let $C$ be a rectifiable Jordan curve, and let $k(\geqq 0)$ be given. Let the function $f(z)$ be analytic interior to $C$, continuous in $\bar{C}$, and let the kth derivative $f^{(k)}(z)=F(s)$ exist on $C$ in the one-dimensional sense, where $s$ denotes arc-length. Let $F(s)$ be continuous on $C$ and satisfy the condition

$$
|F(s+h)+F(s-h)-2 F(s)| \leqq M|h| ;
$$

then $f(z)$ is said to belong to class $Z_{A}^{k}$ on $\dot{C}\left({ }^{5}\right)$.

Of course $f^{(0)}(z)$ indicates the original function $f(z)$. We denote the class $Z_{A}^{0}$ by $Z_{A}$.

It follows [Walsh, Sewell, and Elliott 1, Theorem 2.2] that if $f(z) \in Z_{A}^{k}$ on a rectifiable Jordan curve $C$ whose arc and chord are infinitesimals of the same order (in the sense that $\Delta s / \Delta z$ is uniformly bounded on $C$ ) that $f^{(k)}(z)$ exists and is continuous on $\bar{C}$ in the two-dimensional sense.

The behavior of functions of class $Z_{A}^{k}$ under differentiation and integration is immediately verified - the single-valuedness of the indefinite integral of $f(z)$ on $\bar{C}$ is a consequence of a general form of Cauchy's integral theorem-

THEOREM 1.2. Let $C$ be a rectifiable Jordan curve whose arc and chord are infinitesimals of the same order; if $f(z) \in Z_{A}^{k}, k \geqq 1$, on $C$ then $f^{\prime}(z) \in Z_{A}^{k-1}$ on $C$; if $f(z) \in Z_{A}^{\mathbf{k}}$ on $C, k \geqq 0$, then the indefinite integral of $f(z)$ belongs to $Z_{A}^{k+1}$ on $C$.

To be explicit as to our results, in $\$ 2$ we study the intrinsic properties of functions of class $Z_{A}^{k}$ on the unit circle, with reference to their real and pure imaginary parts, and also study the extension of (1.2) to the closed region $|z| \leqq 1$. In $\$ 3$ we study these properties of class $Z_{A}^{k}$ on a more general curve $C$. In $\$ 4$ we show for $C$ an analytic Jordan curve that $f(z)$ can be uniformly

(4) In considering a harmonic function or harmonic polynomial the qualification real is understood. A polynomial in $z$ of degree $n$ is a function of the form $\sum_{j=0}^{n} a_{j} z^{j}$; a harmonic polynomial of degree $n$ is the real part of such a function.

(5) It is of course possible in Definition 1.1 to require a condition of form (1.2) on $\partial^{k} f(z) / \partial s^{k}$ rather than on $f^{(k)}(z) \equiv F(s)$. This modification of Definition 1.1 would be, however, no great advantage in Theorem 4.1, and would be a disadvantage in proving Theorems 4.4 and 4.5 . The two requirements are equivalent if $C$ is sufficiently smooth; compare Theorem 3.6 and its proof. The letter $k$ will always denote an integer; through Theorem 5.2 we assume $k \geqq 0$. 
approximated in $\bar{C}$ by polynomials in $z$ of degree $n$ with degree of approximation $1 / n^{k+1}$ when and only when $f(z) \in Z_{A}^{k}$ on $C$. Let $G(x, y)$ denote Green's function for the exterior of $C$ with pole at infinity, and let $C_{R}$ denote the level curve $G(x, y)=\log R(>0)$ exterior to $C$. In $\$ 5$ we study the relation between functions of class $Z_{A}^{k}$ on $C_{R}$ and degree of approximation on $\bar{C}$.

We mention for future use other properties of class $\Lambda^{*}$. From Theorem 1.1 Zygmund proves

TheOREM 1.3. A function $F(\theta)$ of class $\Lambda^{*}$ satisfies a Lipschitz condition of arbitrary order $\alpha, 0<\alpha<1$.

It is clear that a function $F(\theta)$ with period $2 \pi$ which satisfies a Lipschitz condition of order unity is of class $\Lambda^{*}$.

Zygmund also establishes

Theorem 1.4. If $F(\theta)$ belongs to $\Lambda^{*}$, so does the function conjugate to $F(\theta)$.

Some further properties of class $\Lambda^{*}$ are needed in the sequel. The class is closed under multiplication:

Theorem 1.5. If the functions $F_{1}(\theta)$ and $F_{2}(\theta)$ belong to class $\Lambda^{*}$, so also does the product $F(\theta) \equiv F_{1}(\theta) \cdot F_{2}(\theta)$.

The function $F(\theta)$ is continuous and possesses the period $2 \pi$. By Theorem 1.1 there exist trigonometric polynomials $T_{1 n}(\theta)$ and $T_{2 n}(\theta)$ of respective degrees $n$ such that for all $\theta,\left|F_{1}(\theta)-T_{1 n}(\theta)\right| \leqq L_{1} / n,\left|F_{2}(\theta)-T_{2 n}(\theta)\right| \leqq L_{2} / n$. The function $F_{2}(\theta)$ is bounded, and the polynomials $T_{1 n}(\theta)$ are uniformly bounded, so we have for all $\theta$

$$
\begin{gathered}
\left|F_{1}(\theta) F_{2}(\theta)-F_{2}(\theta) T_{1 n}(\theta)\right| \leqq L_{3} / n, \quad\left|F_{2}(\theta) T_{1 n}(\theta)-T_{1 n}(\theta) T_{2 n}(\theta)\right| \leqq L_{4} / n, \\
\left|F(\theta)-T_{1 n}(\theta) T_{2 n}(\theta)\right| \leqq\left(L_{3}+L_{4}\right) / n .
\end{gathered}
$$

From the usual formulas for the product of two sines or cosines it follows that the product $T_{1 n}(\theta) T_{2 n}(\theta)$ is a trigonometric polynomial of order $2 n$, so (1.1) follows by Theorem 1.1, and Theorem 1.5 is established.

Theorem 1.6. If $F_{1}(\theta)$ and $F_{2}(\theta)$ belong to class $\Lambda^{*}$, and if $F_{1}(\theta)$ does not vanish, the quotient $F(\theta) \equiv F_{2}(\theta) / F_{1}(\theta)$ also belongs to $\Lambda^{*}$.

By Theorem 1.5 it is sufficient to consider the case $F_{2}(\theta) \equiv 1$. We write

$$
\begin{aligned}
F_{1}(\theta+h) F_{1}(\theta-h) F_{1}(\theta) & {\left[\frac{1}{F_{1}(\theta+h)}+\frac{1}{F_{1}(\theta-h)}-\frac{2}{F_{1}(\theta)}\right] } \\
\equiv & F_{1}(\theta) F_{1}(\theta-h)+F_{1}(\theta) F_{1}(\theta+h)-2 F_{1}(\theta+h) F_{1}(\theta-h) \\
\equiv & F_{1}(\theta)\left[F_{1}(\theta+h)+F_{1}(\theta-h)-2 F_{1}(\theta)\right] \\
& -\left\{\left[F_{1}(\theta+h)\right]^{2}+\left[F_{1}(\theta-h)\right]^{2}-2\left[F_{1}(\theta)\right]^{2}\right\}+\left[F_{1}(\theta+h)-F_{1}(\theta-h)\right]^{2} .
\end{aligned}
$$


Each term of this last member is seen to be in absolute value not greater than $L|h|$, where $L$ is suitably chosen: the first term by the hypothesis (1.1) on $F_{1}(\theta)$; the second term by Theorem 1.5 ; the third term by virtue of Theorem 1.3 with $\alpha=1 / 2$. Theorem 1.6 now follows from the boundedness of $1 / F_{1}(\theta)$.

2. Class $Z_{A}^{k}$. Unit circle. In the definition of class $Z_{A}^{k}$ where $C$ is the unit circle, condition (1.2) is equivalent to an asymptotic condition on $f^{(k+2)}(z)$ :

TheOREm 2.1. A necessary and sufficient condition that $f(z) \in Z_{A}^{k}$ on $|z|=1$ is that $f(z)$ be analytic in $|z|<1$ and $\left(z=r e^{i \theta}\right)$

$$
\left|f^{(k+2)}(z)\right| \leqq L /(1-r), \quad|z|<1 .
$$

Set $f^{(k)}(z)=U(z)+i V(z)$. If $f(z) \in Z_{A}^{k}$ on $|z|=1$, then $U\left(e^{i \theta}\right)$ and $V\left(e^{i \theta}\right)$ belong to class $\Lambda^{*}$. Hence [Zygmund 1, Theorem 13] we have for $0<r<1$

$$
\left|\frac{\partial^{2} f^{(k)}\left(r e^{i \theta}\right)}{\partial \theta^{2}}\right| \leqq\left|\frac{\partial^{2} U\left(r e^{i \theta}\right)}{\partial \theta^{2}}\right|+\left|\frac{\partial^{2} V\left(r e^{i \theta}\right)}{\partial \theta^{2}}\right| \leqq \frac{L_{1}}{1-r} .
$$

Furthermore, $f^{(k+2)}(z)=-\left(1 / z^{2}\right)\left(\partial^{2} f^{(k)} / \partial \theta^{2}-i \partial f^{(k)} / \partial \theta\right)$. From the boundedness of $f^{(k)}(z)$ in $|z| \leqq 1$ we obtain [Sewell 1, Theorem 5.2.4] $\left|\partial f^{(k)}\left(r e^{i \theta}\right) / \partial \theta\right|$ $\leqq L_{2} /(1-r)$. Hence we have

$$
\begin{aligned}
& \left|f^{(k+2)}(z)\right| \leqq\left(L_{1}+L_{2}\right) / r^{2}(1-r), \\
& 0<r<1 \text {; } \\
& \left|f^{(k+2)}(z)\right| \leqq L_{3} /(1-r), \\
& 0<r<1 \text {. }
\end{aligned}
$$

Conversely, suppose $f(z)$ is analytic in $|z|<1$ and that inequality (2.1) is satisfied. Inequality (2.1) implies $\left|f^{(k+1)}(z)\right| \leqq L_{4} /(1-r), r<1$. Thus from the equation $\partial^{2} f^{(k)} / \partial \theta^{2}=-z^{2} f^{(k+2)}(z)-z f^{(k+1)}(z)$, we obtain $\left|\partial^{2} f^{(k)} / \partial \theta^{2}\right|$ $\leqq L_{5} /(1-r)$. The sufficiency of condition (2.1) follows from Zygmund [1, Theorem 13] applied to $\partial^{2} U / \partial \theta^{2}$ and $\partial^{2} V / \partial \theta^{2}$.

We make now

DEFINITION 2.1. Let $s$ denote arc-length measured along a rectifiable Jordan curve $C$. The function $u(z)$ is said to belong to class $Z^{k}$ on $C$ if $u(z)$ is harmonic interior to $C$, continuous in $\bar{C}$, and $\partial^{k} u / \partial s^{k}=U(s)$ exists, is continuous on $C$, and satisfies on $C$ the condition

$$
|U(s+h)+U(s-h)-2 U(s)| \leqq L|h| .
$$

We denote the class $Z^{0}$ by $Z$.

To relate the classes $Z^{k}$ and $Z_{A}^{k}$ for the case that $C$ is the unit circle we prove

ThEOREM 2.2. A necessary and sufficient condition that $f(z)=u(z)+i v(z)$ belong to class $Z_{A}^{k}$ on $|z|=1$ is that $u(z)$ belong to class $Z^{k}$ on $|z|=1$.

For $k=0$, the necessity of the condition is immediate, by separation of $f(z)$ into real and pure imaginary parts. In proving the sufficiency, it follows from Theorem 1.3 that $u(z)$ satisfies a Lipschitz condition of order $\alpha, 0<\alpha<1$, 
on $|z|=1$, then from a well known theorem due to Privaloff that $v(z)$ is continuous $\left({ }^{6}\right)$ in $|z| \leqq 1$, and finally from Theorem 1.4 that $v(z) \in \Lambda^{*}$ on $|z|=1$; thus $f(z) \in Z_{A}$.

For $k \geqq 1$, the necessity of the condition follows at once from the equa$\operatorname{tion}\left({ }^{7}\right) \partial^{k} u(z) / \partial \theta^{k}=\Re\left[\sum_{j=1}^{k} c_{j} z^{j} f^{(j)}(z)\right],|z|=1$, where the $c_{j}$ are constants, since [Walsh, Sewell, and Elliott 1, Theorem 2.1] $f^{(j)}(z), 0 \leqq j \leqq k-1$, satisfies a Lipschitz condition of order unity on $|z|=1$. The function $\partial^{k} v / \partial \theta^{k}$ is conjugate to $\partial^{k} u / \partial \theta^{k}$; thus if $u \in Z^{k}$ on $|z|=1$, then $v \in Z^{k}$ on $|z|=1$. Hence the sufficiency follows from the equation $f^{(k)}(z)=z^{-k} \sum_{j=1}^{k} d_{j}\left(\partial^{j} f / \partial \theta^{j}\right),|z|=1$, the $d_{j}$ being constants, since $\partial^{j} f / \partial \theta^{j}, 0 \leqq j \leqq k-1$, satisfies a Lipschitz condition of order unity.

For the unit circle condition (1.2) is equivalent to the corresponding condition in the closed region:

TheOREM 2.3. Let $f(z)$ be analytic interior to $\gamma:|z|=1$ and continuous on $\bar{\gamma} . A$ necessary and sufficient condition that $f(z) \in Z_{A}$ on $\gamma$ is

$$
|f(z)+f(\zeta)-2 f[(z+\zeta) / 2]| \leqq L|z-\zeta|, \quad|z| \leqq 1, \quad|\zeta| \leqq 1 .
$$

To prove the necessity of the condition we establish (2.3) first for both $z$ and $\zeta$ on $\gamma$ :

$$
\begin{aligned}
\left|\frac{f\left(e^{i(\phi+\theta)}\right)+f\left(e^{i(\phi-\theta)}\right)-2 f\left(e^{i \phi} \cos \theta\right)}{\sin \theta}\right| & \\
& \leqq\left|\frac{f\left(e^{i(\phi+\theta)}\right)+f\left(e^{i(\phi-\theta)}\right)-2 f\left(e^{i \phi}\right)}{\theta}\right|\left|\frac{\theta}{\sin \theta}\right| \\
& +2\left|\frac{f\left(e^{i \phi}\right)-f\left(e^{i \phi} \cos \theta\right)}{\theta}\right|\left|\frac{\theta}{\sin \theta}\right| \leqq M_{2} ;
\end{aligned}
$$

here we make the choice $0 \leqq \theta \leqq \pi / 2$, which implies the general inequality $2|\theta| \leqq \pi|\sin \theta|$, and we make use of (1.2); by Theorem $1.3, f(z)$ satisfies on $\gamma$ a Lipschitz condition of order $3 / 4$, so [Sewell 1 , Theorem 1.2.7] $f(z)$ also satisfies a Lipschitz condition of order $3 / 4$ in $\bar{\gamma}$ :

$$
\begin{aligned}
\left|f\left(e^{i \phi}\right)-f\left(e^{i \phi} \cos \theta\right)\right| & \leqq M_{1}|1-\cos \theta|^{3 / 4}=M_{1}\left[2 \sin ^{2}(\theta / 2)\right]^{3 / 4} \\
& \leqq 2^{-3 / 4} M_{1}|\theta|^{3 / 2} .
\end{aligned}
$$

We next establish (2.3) for $\zeta$ fixed on $\gamma$ but $z$ interior to $\gamma$. The function

$$
F_{\zeta}(z) \equiv \frac{f(z)+f(\zeta)-2 f[(z+\zeta) / 2]}{z-\zeta}
$$

(6) If two functions are conjugate in a region and continuous in the corresponding closed region, we say that the functions are conjugate in the closed region.

$\left(^{7}\right) \Re[F(z)]$ denotes the real part of $F(z) ; \Im[F(z)]$ denotes the imaginary part of $F(z)$ divided by $i$. 
is analytic for $|z|<1$, and continuous for $|z| \leqq 1$ except for $z=\zeta$. This function is bounded for $z$ on $\gamma$, as we have just shown, except that $F_{\zeta}(\zeta)$ is not defined. By the Lipschitz condition of order $\alpha(0<\alpha<1)$ on $f(z)$ in $\bar{\gamma}$ we have $(|z|<1)$

$$
\begin{gathered}
|f(z)-f[(z+\zeta) / 2]| \leqq L_{1}|z-\zeta|^{\alpha} \\
|f(\zeta)-f[(z+\zeta) / 2]| \leqq L_{1}|z-\zeta|^{\alpha}, \quad\left|F_{\zeta}(z)\right| \leqq L_{2}|z-\zeta|^{\alpha-1}
\end{gathered}
$$

It is a consequence of this last inequality and the Phragmén-Lindelöf principle [for example, Nevanlinna 1, p. 43] that a bound of $F_{\zeta}(z)$ for $z$ on $\gamma$ $(z \neq \zeta)$ is also a bound for $z$ in $\bar{\gamma}$; this bound (say $B$ ) can be chosen to be independent of $\zeta$.

To continue the proof of Theorem 2.3 we consider $F_{\zeta}(z)$ for $z$ fixed interior to $\gamma$; then $F_{\zeta}(z)$ is an analytic function of $\zeta$ for $\zeta$ interior to $\gamma$, even in the point $\zeta=z$ if suitably defined there; moreover $F_{\zeta}(z)$ is continuous for $\zeta$ in $\bar{\gamma}$. We have just proved $\left|F_{\zeta}(z)\right| \leqq B$ for $\zeta$ on $\gamma$, so this same inequality is valid for $\zeta$ in $\bar{\gamma}$, and this completes the proof of the necessity of condition (2.3).

Conversely, if condition (2.3) is satisfied, then $f(z) \in Z_{A}$ on $\gamma$, for

$$
\begin{aligned}
&\left|\frac{f\left(e^{i(\phi+\theta)}\right)+f\left(e^{i(\phi-\theta)}\right)-2 f\left(e^{i \phi}\right)}{\theta}\right| \\
& \leqq\left|\frac{f\left(e^{i(\phi+\theta)}\right)+f\left(e^{i(\phi-\theta)}\right)-2 f\left(e^{i \phi} \cos \theta\right)}{\sin \theta}\right|\left|\frac{\sin \theta}{\theta}\right| \\
&+2\left|\frac{f\left(e^{i \phi}\right)-f\left(e^{i \phi} \cos \theta\right)}{\theta}\right| \leqq M_{3} ;
\end{aligned}
$$

the inequality $|(\sin \theta) / \theta| \leqq 1$ together with the hypothesis implies the boundedness of the first term in the second member; from (2.3) it follows that $f\left(r e^{i \phi}\right)$ satisfies a condition of form (1.2) on $0 \leqq r \leqq 1$, and hence by Theorem 1.3 (see below) the function $f\left(r e^{i \phi}\right)$ satisfies a Lipschitz condition of order $3 / 4$ in $r$; we note on examining Zygmund's proof of Theorem 1.3 that the constant involved in the Lipschitz condition may be chosen to be independent of $\phi$; thus

$$
\left|f\left(e^{i \phi}\right)-f\left(e^{i \phi} \cos \theta\right)\right| \leqq M_{4}|1-\cos \theta|^{3 / 4} \leqq M_{5}|\theta|^{3 / 2} \text {. }
$$

Hence Theorem 2.3 is established.

This application of Theorem 1.3, to show that $f\left(r e^{i \phi}\right)$ satisfies a Lipschitz condition in $r$, involves the as yet unproved fact that if a continuous function $F(\theta)$ satisfies condition (1.1) uniformly on $0 \leqq \theta \leqq \theta_{0}$, then $F(\theta)$ satisfies there a Lipschitz condition of arbitrary order $\alpha(0<\alpha<1)$. In the proof we assume $\theta_{0}=\pi, F(0)=F(\pi)=0$; this involves no loss of generality, for hypothesis and conclusion are not effectively altered if we add to $F(\theta)$ a suitable linear func- 
tion of $\theta$. We introduce the definition $F(-\theta) \equiv-F(\theta), 0 \leqq \theta \leqq \pi$. Given $0 \leqq \theta \leqq \pi / 2,0 \leqq \delta \leqq \pi / 2, \theta \leqq \delta$, we have by (1.1) and the equation $F(0)=0$,

$$
\begin{aligned}
|F(\delta-\theta)+F(\delta+\theta)-2 F(\delta)| & \leqq M \theta, \\
|F(2 \delta-2 \theta)-2 F(\delta-\theta)| & \leqq M(\delta-\theta), \\
|F(2 \theta)-2 F(\theta)| & \leqq M \theta, \\
|-F(2 \theta)-F(2 \delta-2 \theta)+2 F(\delta)| & \leqq M|\delta-2 \theta|,
\end{aligned}
$$

whence by the equation $F(\delta-\theta)=-F(\theta-\delta)$,

$$
|F(\theta+\delta)+F(\theta-\delta)-2 F(\theta)| \leqq M[2 \theta+(\delta-\theta)+|\delta-2 \theta|] .
$$

In the case $2 \theta \leqq \delta$, the second member of (2.4) reduces to $M(2 \delta-\theta)$, which is not greater than $2 M \delta$; in the case $\theta \leqq \delta<2 \theta$ the second member of (2.4) reduces to $3 M \theta$, which is not greater than $3 M \delta$. Thus the extended $F(\theta)$ satisfies in the interval $-\pi \leqq \theta \leqq \pi$ a condition of form (1.1) with $M$ replaced by $3 M$, for $0 \leqq \delta \leqq \pi / 2$. It then follows readily that if $F(\theta)$ is further extended so as to possess the period $2 \pi$, this final $F(\theta)$ is of class $\Lambda^{*}$; Theorem 1.3 applies. Incidentally, it follows now by addition of a suitable broken-line function of class $\Lambda^{*}$ that the original $F(\theta)$ defined merely in the interval $0 \leqq \theta \leqq \pi$ but without assuming $F(0)=F(\pi)=0$ can be extended so as to be of class $\Lambda^{*}$.

Zygmund [1, p. 64] relates condition (1.2) to higher differences. From his results we may conclude: Let $f(z)$ be analytic in $|z|<1$ and continuous in $|z| \leqq 1$. A necessary and sufficient condition that $f(z) \in Z_{A}^{k}$ on $|z|=1$ is that $\left|F[\theta+(k+2) h]-C_{k+2,1} F[\theta+(k+1) h]+\cdots+(-1)^{k+2} F(\theta)\right| \leqq L|h|^{k+1}$, where $f\left(e^{i \theta}\right)=F(\theta)$.

From Theorems 1.5 and 1.6 it follows directly that when $C$ is the unit circle the class $Z_{A}$ is closed under multiplication and is closed under division by nonvanishing functions; we do not emphasize these results here, for they apply (Theorem 3.3) also to more general Jordan curves $C$.

3. Class $Z_{A}^{k}$. More general curves. We establish in this section properties of functions which belong to the class $Z_{A}^{\boldsymbol{k}}$ on a curve more general than the unit circle.

Theorem 3.1. In Definition 1.1, inequality (1.2) is equivalent to the inequality

$$
\left|F\left(s+h_{1}\right)+F\left(s-h_{2}\right)-2 F(s)\right| \leqq M_{1}\left|h_{1}\right|,
$$

for all $h_{1}$ and $h_{2}$ with $\left|h_{1}-h_{2}\right|^{\alpha} \leqq L_{\alpha}\left|h_{1}\right|, 0<\alpha<1$, where $M_{1}$ and $L_{\alpha}$ are suitably chosen.

Condition (1.2) is an obvious consequence of (3.1), even if we assume (3.1) merely for a single value of $\alpha$; we proceed to show that if $\alpha$ and $L_{\alpha}$ are given, then $M_{1}$ depending on $L_{\alpha}$ exists so that (1.2) implies (3.1) if $\left|h_{1}-h_{2}\right| \alpha \leqq L_{\alpha}\left|h_{1}\right|$. 
It follows from Theorem 1.3 and (1.2) that $F(s)$ satisfies on $C$ a Lipschitz condition of order $\alpha:\left|F\left(s_{1}\right)-F\left(s_{2}\right)\right| \leqq M_{\alpha}\left|s_{1}-s_{2}\right| \alpha$, whence $\left|F\left(s-h_{2}\right)-F\left(s-h_{1}\right)\right|$ $\leqq M_{\alpha}\left|h_{1}-h_{2}\right|^{\alpha}$. We have

$$
\begin{aligned}
\mid F\left(s+h_{1}\right)+ & F\left(s-h_{2}\right)-2 F(s) \mid \\
& \leqq\left|F\left(s+h_{1}\right)+F\left(s-h_{1}\right)-2 F(s)\right|+\left|F\left(s-h_{2}\right)-F\left(s-h_{1}\right)\right| \\
& \leqq M\left|h_{1}\right|+L_{\alpha} M_{\alpha}\left|h_{1}\right|,
\end{aligned}
$$

so (3.1) follows if we choose $M_{1}=M+L_{\alpha} M_{\alpha}$.

We study next the invariance of (1.2) under transformation:

THEOREM 3.2. Let $f(x)$, continuous and periodic with period a, satisfy a condition of form (1.2). Set $f(x) \equiv F(y)$, where $x=x(y)$ is a one-to-one transformation of the interval $0 \leqq y \leqq b$ onto the interval $0 \leqq x \leqq a$ for which $x^{\prime}(y)$ exists and satisfies a Lipschitz condition of some positive order $\alpha$. We assume $x(0)=0$, $x(y+b) \equiv x(y)+a$. Then $F(y)$ satisfies a condition of form (1.2) with respect to $y$.

We write

$$
\begin{aligned}
& \left|\frac{F(y \mid l)+F(y-h)-2 F(y)}{h}\right|=\left|\frac{f[x(y+h)]+f[x(y-h)]-2 f[x(y)]}{h}\right| \\
& \leqq\left|\frac{f[x(y+h)]+f[x(y-h)]-2 f([x(y+h)+x(y-h)] / 2)}{[x(y+h)-x(y-h)] / 2}\right| \\
& \cdot\left|\frac{x(y+h)-x(y-h)}{2 h}\right| \\
& +2\left|\frac{f([x(y+h)+x(y-h)] / 2)-f[x(y)]}{h}\right| .
\end{aligned}
$$

Since $x^{\prime}(y)$ is continuous, $x(y)$ satisfies a Lipschitz condition of order unity; hence it follows from the hypothesis on $f(x)$ that the first term in the last member of (3.2) is bounded. Furthermore, $f(x)$ satisfies by Theorem 1.3 a Lipschitz condition of order $1 /(1+\alpha)$. Thus (we use the Lipschitz condition on $x^{\prime}(s)$ )

$$
\begin{aligned}
\left|\frac{f([x(y+h)+x(y-h)] / 2)-f[x(y)]}{h}\right| \\
\leqq L \frac{|x(y+h)+x(y-h)-2 x(y)|^{1 /(1+\alpha)}}{|h|} \\
\leqq \frac{L}{|h|}\left|\int_{y}^{y+h}\left[x^{\prime}(\zeta)-x^{\prime}(\zeta-h)\right] d \zeta\right|^{1 /(1+\alpha)} \leqq L L_{1} .
\end{aligned}
$$

Hence Theorem 3.2 follows. 
Since $f(x)$ satisfies a Lipschitz condition of order $(2+\alpha) /(2+2 \alpha)$ we obtain similarly: In addition to the hypothesis of Theorem 3.2 suppose $[f(x+h)$ $+f(x-h)-2 f(x)] / h$ approaches zero uniformly as $h$ approaches zero. Then $[F(y+h)+F(y-h)-2 F(y)] / h$ approaches zero uniformly as $h$ approaches zero.

The class $Z_{A}$ is closed under multiplication, and under division by a nonvanishing function of the class:

TheOREM 3.3. Let $C$ be a rectifiable Jordan curve. Suppose $f(z)$ and $g(z)$ belong to the class $Z_{A}$ on $C$. Then $F_{1}(z)=f(z) \cdot g(z)$ belongs to the class $Z_{A}$ on $C$. Furthermore, if $f(z) \neq 0$ in $\bar{C}$, then $F_{2}(z)=[g(z) / f(z)]$ belongs to the class $Z_{A}$ on $C$.

The analyticity interior to $C$ and continuity in $\bar{C}$ of $F_{1}(z)$ and $F_{2}(z)$ are clear. That $F_{1}(z)$ and $F_{2}(z)$, when expressed as functions of arc-length $s$, satisfy a condition of form (1.2) follows from Theorems 1.5 and 1.6.

Let $C$ be a Jordan curve lying in the $z$-plane and let $z=\chi(w)$ map $|w|<1$ one-to-one and conformally onto the interior of $C$. With this notation we introduce

Definition 3.1. A curve $C$ is said to be of Type $\mathrm{G}$ if $C$ is a Jordan curve such that $\chi^{\prime}(w)$ exists, is different from zero, and satisfies a Lipschitz condition of some positive order $\alpha$ on $|w|=1$.

Definition 3.1 is independent of the particular mapping function $\chi(w)$ chosen.

The class $Z_{A}^{k}$ is invariant under suitable conformal transformation:

THEOREM 3.4. Let $C$ be a curve of Type G; suppose $\chi(w) \in Z_{A}^{k}$ on $|w|=1$. If $f(z) \in Z_{A}^{k}$ on $C$, then $F(w) \in Z_{A}^{k}$ on $|w|=1$, where $F(w)=f[\chi(w)]$. Conversely, if $F(w) \in Z_{A}^{k}$ on $|w|=1$, then $f(z) \in Z_{A}^{k}$ on $C$.

Set $w=r e^{i \theta}$; let $s$ denote arc-length measured along $C$; let $s=s(\theta), \theta=\theta(s)$. Since $d s(\theta) / d \theta=\left|\chi^{\prime}(w)\right|$ and $d \theta(s) / d s=1 /\left|\chi^{\prime}(w)\right|$, from the hypothesis on $C$ it follows that $s^{\prime}(\theta)$ and $\theta^{\prime}(s)$ exist and satisfy a Lipschitz condition of some positive order $\alpha$. Hence for the case $k=0$, Theorem 3.4 is an immediate consequence of Theorem 3.2.

Suppose $k \geqq 1$. Let $f(z) \in Z_{A}^{k}$ on $C$. By Theorem 3.2 the function $d^{k} f\left[\chi\left(e^{i \theta}\right)\right] / d z^{k}$ satisfies a condition of form (1.2) in $\theta$. From the uniform boundedness on $C$ of $\left|f^{(k)}(z)\right|$ and the hypothesis on $C$ we infer that $f^{(j)}(z)$, $0 \leqq j \leqq k-1$, satisfies on $|w|=1$ a Lipschitz condition in $\theta$ of order unity and hence a condition in $\theta$ of form (1.2); for example we have with $z_{j}=\chi\left(e^{i \theta_{j}}\right)$ :

$$
\begin{aligned}
\left|f^{(k-1)}\left(z_{1}\right)-f^{(k-1)}\left(z_{2}\right)\right| & \leqq \int_{z_{1}}^{z_{2}}\left|f^{(k)}(z)\right| d s \\
& \leqq M \int_{\theta_{2}}^{\theta_{1}}\left|\chi^{\prime}(w)\right| d \theta \leqq M M_{1}\left|\theta_{1}-\theta_{2}\right|
\end{aligned}
$$

The first half of Theorem 3.4 now follows from Theorem 3.3 and the equations 


$$
\begin{aligned}
F^{\prime}(w) & =f^{\prime}(z) \cdot \chi^{\prime}(w) \\
F^{\prime \prime}(w) & =f^{\prime \prime}(z)\left[\chi^{\prime}(w)\right]^{2}+f^{\prime}(z) \cdot \chi^{\prime \prime}(w),
\end{aligned}
$$

The proof for $k \geqq 1$ of the second part of Theorem 3.4 is analogous and will be omitted.

We next extend Theorem 2.1. Let $w=\Omega(z)$ be the function inverse to $z=\chi(w)$. Let $C_{r}$ denote the curve $|\Omega(z)|=r, 0<r<1$, interior to $C$.

Theorem 3.5. Let $C$ be a curve of Type G. A necessary and sufficient condition that $f(z) \in Z_{A}^{k}$ on $C$ is that $f(z)$ be analytic interior to $C$ and

$$
\left|f^{(k+2)}(z)\right| \leqq L /(1-r), \quad z \text { on } C_{r}, 0<r<1 .
$$

Set $d^{k} f[\chi(w)] / d z^{k}=F(w)$; suppose $w=r e^{i \theta}$. If $f(z) \in Z_{A}^{k}$ on $C$ then by Theorem 3.4, $F(w) \in Z_{A}$ on $|w|=1$. Hence by Theorem 2.1 we have $\left|F^{\prime \prime}(w)\right|$ $\leqq M /(1-r)$. It follows that $\left|F^{\prime}(w)\right| \leqq M_{1}+M|\log (1-r)| \leqq M_{2}(1-r)^{-\alpha}$, where $\alpha$ is the constant of Definition 3.1. Furthermore, by a result due to Hardy and Littlewood [see, for example, Sewell 1, Theorem 1.2.1] we have $\left|\chi^{\prime \prime}(w)\right| \leqq M_{3}(1-r)^{\alpha-1}$. Thus (3.3) follows from the equation $f^{(k+2)}(z)$ $=F^{\prime \prime}(w)\left[\chi^{\prime}(w)\right]^{-2}-F^{\prime}(w) \chi^{\prime \prime}(w)\left[\chi^{\prime}(w)\right]^{-3}$.

Now suppose (3.3) is satisfied; for $z$ on $C_{r}$ we have

$$
\begin{aligned}
\left|f^{(k+1)}(z)\right| & \leqq\left|f^{(k+1)}[\chi(0)]\right|+\int_{\chi(0)}^{z}\left|f^{(k+2)}(z)\right||d z| \\
& \leqq L_{1}+L \int_{0}^{r}\left[\left|\chi^{\prime}\left(\rho e^{i \theta}\right)\right| /(1-\rho)\right] d \rho \\
& \leqq L_{2}|\log (1-r)| \leqq L_{3}(1-r)^{-\alpha} .
\end{aligned}
$$

From the equation $F^{\prime \prime}(w)=f^{(k+2)}(z)\left[\chi^{\prime}(w)\right]^{2}+f^{(k+1)}(z) \chi^{\prime \prime}(w),|w|<1$, it thus follows that $\left|F^{\prime \prime}(w)\right| \leqq L_{4} /(1-r)$. Hence by Theorem 2.1 we have $F(w) \in Z_{A}$ on $|w|=1$, and by Theorem 3.4 we have $f^{(k)}(z) \in Z_{A}$ on $C$; thus $f(z) \in Z_{A}^{k}$ on $C$.

We consider next the relation between the classes $Z^{k}$ and $Z_{\boldsymbol{A}}^{k}$.

Theorem 3.6. Let $C$ be an analytic Jordan curve. A necessary and sufficient condition that $f(z)=u(z)+i v(z)$ belong to class $Z_{A}^{k}$ on $C$ is that $u(z)$ belong to class $Z^{k}$ on $C$.

Suppose $k=0$. The necessity of the condition is then obvious. Conversely, if $u(z) \in Z$ on $C$, it follows from Theorem 3.2 that $u[\chi(w)] \in Z$ on $|w|=1$. Hence by Theorem 2.2, $f[\chi(w)] \in Z_{A}$ on $|w|=1$, and thus by Theorem 3.2, $f(z) \in Z_{A}$ on $C$.

Now suppose $k \geqq 1$. Let the equation of $C$ in terms of arc-length $s$ be $z=\tau(s)$. If $f(z) \in Z_{\boldsymbol{A}}^{k}$ on $C$, then all the functions $f^{(j)}(z)$ and $\tau^{(j+1)}(s)$ 
$(j=0,1, \cdots, k-1)$ satisfy a Lipschitz condition in $s$ of order unity and hence a condition of form (1.2) on $C$. Thus from the equations

$$
\begin{aligned}
\frac{\partial f}{\partial s} & =f^{\prime}(z) \cdot \tau^{\prime}(s), \\
\frac{\partial^{2} f}{\partial s^{2}} & =f^{\prime \prime}(z)\left[\tau^{\prime}(s)\right]^{2}+f^{\prime}(z) \cdot \tau^{\prime \prime}(s),
\end{aligned}
$$

and from Theorem 3.3 it follows that $\partial^{k} f / \partial s^{k}$, and hence $\partial^{k} u / \partial s^{k}$, satisfies a condition of form (1.2).

Conversely, if $u(z) \in Z^{k}, k \geqq 1$, then by Theorem $3.2, \partial^{k} u[\chi(w)] / \partial s^{k} \in Z$ on $|w|=1$. Hence from the equations

$$
\begin{aligned}
& \frac{\partial u}{\partial \theta}=\frac{\partial u}{\partial s} \cdot \frac{d s}{d \theta} \\
& \frac{\partial^{2} u}{\partial \theta^{2}}=\frac{\partial^{2} u}{\partial s^{2}}\left(\frac{d s}{d \theta}\right)^{2}+\frac{\partial u}{\partial s} \cdot \frac{d^{2} s}{d \theta^{2}}
\end{aligned}
$$

we obtain $u(z) \in Z^{k}$ on $|w|=1$. Thus by Theorem 2.2 it follows that $f[\chi(w)]$ $\in Z_{A}^{k}$ on $|w|=1$; application of Theorem 3.4 completes the proof.

We note that the proof of Theorem 3.6 requires much less than the analyticity of $C$. It is sufficient that $C$ be of Type $G$ and that $\tau^{(k)}(s), d^{k} s / d \theta^{k}$ be continuous and satisfy a condition of form (1.2).

Theorem 3.7. Let $C$ be a curve of $T y p e \mathrm{G}$; let $f(z) \in Z_{A}$ on $C$. Then if $z_{1}, z_{2}$, and $\left(z_{1}+z_{2}\right) / 2$ lie in $\bar{C}$ we have

$$
\left|\frac{f\left(z_{1}\right)+f\left(z_{2}\right)-2 f\left[\left(z_{1}+z_{2}\right) / 2\right]}{z_{1}-z_{2}}\right| \leqq L .
$$

Set $f[\chi(w)]=F(w) ; z_{k}=\chi\left(w_{k}\right)$. If $z_{1}, z_{2}$, and $\left(z_{1}+z_{2}\right) / 2$ lie in $\bar{C}$, we have

$$
\begin{aligned}
& \left|\frac{f\left(z_{1}\right)+f\left(z_{2}\right)-2 f\left[\left(z_{1}+z_{2}\right) / 2\right]}{z_{1}-z_{2}}\right| \\
& \leqq\left|\frac{w_{1}-w_{2}}{z_{1}-z_{2}}\right| \cdot\left\{\left|\frac{F\left(w_{1}\right)+F\left(w_{2}\right)-2 F\left[\left(w_{1}+w_{2}\right) / 2\right]}{w_{1}-w_{2}}\right|\right. \\
& \left.+2\left|\frac{f\left[\left(z_{1}+z_{2}\right) / 2\right]-f\left[\chi\left(\left(w_{1}+w_{2}\right) / 2\right)\right]}{w_{1}-w_{2}}\right|\right\} .
\end{aligned}
$$

From the hypothesis on $C$ we have $\left|w_{1}-w_{2}\right| /\left|z_{1}-z_{2}\right| \leqq L_{1}$; by Theorems 3.4 and 2.3 the first term in the braces is bounded; by Theorem 1.3 the function 
$f(z)$ satisfies on $C$ a Lipschitz condition in $s$ and hence in $z$ of order $1 /(1+\alpha)$, where $\alpha$ is the constant of Definition 3.1; then [Sewell 1, Theorem 1.2.7] the function $f(z)$ satisfies a Lipschitz condition of order $1 /(1+\alpha)$ in $\bar{C}$. Hence the second term in braces is not greater than

$$
L_{2}\left|\chi\left(w_{1}\right)+\chi\left(w_{2}\right)-2 \chi\left[\left(w_{1}+w_{2}\right) / 2\right]\right| 1 /(1+\alpha) /\left|w_{1}-w_{2}\right|
$$

But

$$
\begin{aligned}
\left|\chi\left(w_{1}\right)+\chi\left(w_{2}\right)-2 \chi\left[\left(w_{1}+w_{2}\right) / 2\right]\right| & \\
\quad & \leqq \int_{\left(w_{1}+w_{2}\right) / 2}^{w_{2}}\left|\chi^{\prime}(\zeta)-\chi^{\prime}\left[\zeta-\left(w_{2}-w_{1}\right) / 2\right]\right||d \zeta| \\
& \leqq M_{1} \int_{\left(w_{1}+w_{2}\right) / 2}^{w_{2}}\left|w_{2}-w_{1}\right| \alpha|d \zeta| \leqq M_{2}\left|w_{1}-w_{2}\right|^{1+\alpha}:
\end{aligned}
$$

Theorem 3.7 now follows.

In the converse direction we prove

THEOREM 3.8. Let $C$ be a Jordan curve with equation $z=\tau(s)$, where $s$ denotes arc-length measured along $C$. Suppose $\tau^{\prime}(s)$ exists and satisfies a Lipschitz condition of order unity. Let $f(z)$ be analytic interior to $C$ and continuous in $\bar{C}$. Suppose

$$
\left|\frac{f\left(z_{1}\right)+f\left(z_{2}\right)-2 f\left[\left(z_{1}+z_{2}\right) / 2\right]}{z_{1}-z_{2}}\right| \leqq L,
$$

provided $z_{1}, z_{2}$, and $\left(z_{1}+z_{2}\right) / 2$ lie in $\bar{C}$. Then $f(z) \in Z_{A}$ on $C$.

Let $M>1$ be a constant such that $\left|\tau^{\prime}\left(s_{1}\right)-\tau^{\prime}\left(s_{2}\right)\right| \leqq M\left|s_{1}-s_{2}\right|$. Let $\omega(s)$ denote the angle which the positively directed tangent to $C$ at $z=\tau(s)$ makes with the $x$-axis. Then $\omega(s)$ satisfies a Lipschitz condition of order unity in $s$, for we have $\left|\tau^{\prime}(s)\right|=1, \omega(s)=-i \log \tau^{\prime}(s)$, and hence if $|\Delta s| \leqq 1 / 2 M$ we have

$$
\begin{aligned}
& |\omega(s+\Delta s)-\omega(s)|=\left|\log \frac{\tau^{\prime}(s+\Delta s)}{\tau^{\prime}(s)}\right| \\
& \quad \leqq\left|\frac{\tau^{\prime}(s+\Delta s)-\tau^{\prime}(s)}{\tau^{\prime}(s)}\right|\left[1+\frac{1}{2}\left|\frac{\tau^{\prime}(s+\Delta s)-\tau^{\prime}(s)}{\tau^{\prime}(s)}\right|+\cdots\right] \\
& \leqq M|\Delta s| \sum_{n=1}^{\infty} 1 / n 2^{n-1} \leqq 2 M|\Delta s|
\end{aligned}
$$

Hence it follows [Seidel 1, p. 217] that there exists a constant $\delta_{0}, 0<\delta_{0} \leqq 1$, such that through each point $P$ of $C$ there is a circle tangent to $C$ at $P$ with radius $\delta_{0}$, whose closed interior lies in $\bar{C}$. It is clearly sufficient to prove inequality (1.2) for $h$ sufficiently small. We henceforth assume $0 \leqq h \leqq \delta_{0} / 4 M$. 
The point $\zeta$ on the inner normal to $C$ at $\tau(s)$ which is such that $|\zeta-\tau(s)|=\eta$ is $\zeta=\tau(s)+i \eta \tau^{\prime}(s)$. Set $a_{\mu}=\tau(s-h)+\mu 2 M h i \tau^{\prime}(s-h), b_{\mu}=\tau(s)+\mu 2 M h i \tau^{\prime}(s)$, $c_{\mu}=\tau(s+h)+\mu 2 M h i \tau^{\prime}(s+h)$, where $\mu=0,1,2$. The circle $\Gamma ;\left|z-b_{2}\right|=4 M h$ is tangent to $C$ at $\tau(s)$ and lies in $\bar{C}$. The points $a_{1}, a_{2}, c_{1}$, and $c_{2}$ lie in $\bar{\Gamma}$ for

$$
\begin{aligned}
\left|a_{1}-b_{2}\right| & \leqq\left|a_{1}-a_{2}\right|+\left|a_{2}-b_{2}\right| \\
& \leqq 2 M h+|\tau(s)-\tau(s-h)|+4 M h\left|\tau^{\prime}(s)-\tau^{\prime}(s-h)\right| \leqq 4 M h, \\
\left|c_{1}-b_{2}\right| & \leqq\left|c_{1}-c_{2}\right|+\left|c_{2}-b_{2}\right| \\
& \leqq 2 M h+|\tau(s)-\tau(s+h)|+4 M h\left|\tau^{\prime}(s)-\tau^{\prime}(s+h)\right| \leqq 4 M h,
\end{aligned}
$$

since $4 M h \leqq 1$.

Hence we have

$$
\begin{aligned}
&|f[\tau(s+h)]+f[\tau(s-h)]-2 f[\tau(s)]| \\
&=\left|f\left(c_{0}\right)+f\left(a_{0}\right)-2 f\left(b_{0}\right)\right| \leqq\left|f\left(c_{0}\right)+f\left(c_{2}\right)-2 f\left(c_{1}\right)\right| \\
&+\left|f\left(a_{0}\right)+f\left(a_{2}\right)-2 f\left(a_{1}\right)\right|+\left|f\left(a_{2}\right)+f\left(c_{2}\right)-2 f\left[\left(a_{2}+c_{2}\right) / 2\right]\right| \\
&+2\left|f\left(a_{1}\right)+f\left(c_{1}\right)-2 f\left[\left(a_{1}+c_{1}\right) / 2\right]\right|+2\left|f\left(b_{2}\right)+f\left(b_{0}\right)-2 f\left(b_{1}\right)\right| \\
&+2\left|f\left(b_{2}\right)-f\left[\left(a_{2}+c_{2}\right) / 2\right]\right|+4\left|f\left(b_{1}\right)-f\left[\left(a_{1}+c_{1}\right) / 2\right]\right| .
\end{aligned}
$$

The first three terms of the last member are by hypothesis each not greater than $4 L M h$; the fourth and fifth terms are each not greater than $8 L M h$. By Theorems 1.3 and 2.3 the function $f(z)$ satisfies a Lipschitz condition of order $3 / 4$ in the closed region $\left|z-\left[\tau(s)+i \delta_{0} \tau^{\prime}(s)\right]\right| \leqq \delta_{0}$. The Lipschitz constant here can be chosen to be independent of $s$; compare Zygmund's proof of Theorem 1.3. Hence we have

$$
\begin{aligned}
& \left|f\left(b_{2}\right)-f\left[\left(a_{2}+c_{2}\right) / 2\right]\right| \leqq M_{3}\left|b_{2}-\left(a_{2}+c_{2}\right) / 2\right|^{3 / 4} \\
& \left|f\left(b_{1}\right)-f\left[\left(a_{1}+c_{1}\right) / 2\right]\right| \leqq M_{3}\left|b_{1}-\left(a_{1}+c_{1}\right) / 2\right|^{3 / 4} .
\end{aligned}
$$

Furthermore,

$$
\begin{aligned}
2\left|b_{2}-\left(a_{2}+c_{2}\right) / 2\right| \leqq & \tau(s+h)+\tau(s-h)-2 \tau(s) \mid \\
& +4 M h\left[\left|\tau^{\prime}(s)-\tau^{\prime}(s+h)\right|+\left|\tau^{\prime}(s-h)-\tau^{\prime}(s)\right|\right] \\
\leqq & \int_{8}^{8+h}\left|\tau^{\prime}(s)-\tau^{\prime}(s-h)\right| d s+8 M^{2} h^{2} \leqq 9 M^{2} h^{2} ; \\
2\left|b_{1}-\left(a_{1}+c_{1}\right) / 2\right| \leqq & 5 M^{2} h^{2} .
\end{aligned}
$$

The theorem follows.

4. Problem $\alpha$. If $C$ consists of a finite number of mutually exterior rectifiable Jordan curves, we say $u(z) \in Z^{k}\left[f(z) \in Z_{A}^{k}\right]$ on $C$ if $u(z) \in Z^{k}\left[f(z) \in Z_{A}^{k}\right]$ on each component of $C$; we denote by $\bar{C}$ the sum of the closed interiors of these curves. Problem $\alpha$ here is the study of polynomial approximation on $\bar{C}$ to functions which belong to classes $Z^{k}$ and $Z_{A}^{k}$ on $C$. 
THEOREM 4.1. Let $C$ consist of a finite number of mutually exterior analytic Jordan curves. Let $u(z) \in Z^{k}$ on $C$. Set $f(z)=u(z)+i v(z)$ where $v(z)$ is conjugate to $u(z)$. Then there exist polynomials $\pi_{n}(z)$ in $z$ such that

$$
\left|f(z)-\pi_{n}(z)\right| \leqq L / n^{k+1}, \quad \quad z \text { on } \bar{C} .
$$

Here and subsequently we write "there exist polynomials" rather than "for each $n, n=1,2, \cdots$, there exists a polynomial of degree $n$." The degree of a polynomial $\pi_{n}(z), p_{n}(z)$, or $q_{n}(z)$ will always be indicated by its subscript.

By Theorem 3.6, $f(z) \in Z_{A}^{k}$ on $C$. Let $C$ consist of the mutually exterior curves $\Gamma_{\nu}, \nu=1, \cdots, \lambda$; let $z=\chi_{\nu}(w)$ map $|w|<1$ onto the interior of $\Gamma_{\nu}$. Then by Theorem 3.4, $f\left[\chi_{\nu}(w)\right] \in Z_{A}^{k}$ on $|w|=1$. If $w=r e^{i \theta}$, it follows that $\partial^{k} f\left[\chi_{\nu}(w)\right] / \partial \theta^{k}$ satisfies a condition of form (1.1) on $|w|=1$. The results of Zygmund [1, Theorem 7 and §5] together with a method used by Walsh, Sewell, and Elliott [1, Theorem 8.1] now yield the desired conclusion.

In the study of the converse problem we use the following definition:

Definition 4.1. A curve $C$ is said to be of Type D if

(i) $C$ is a rectifiable Jordan curve whose arc and chord are infinitesimals of the same order; and

(ii) there exists a number $\delta_{0}>0$ such that if $P$ is any point of $C$, there is a circle of radius $\delta_{0}$ through $P$ whose closed interior lies in $\bar{C}$.

We shall need the following two theorems [Walsh, Sewell, and Elliott 1, Theorems 7.2, 7.4], of which the first is essentially due to Szegö:

TheOREM 4.2. Let $C$ be a curve of Type D. Let $\pi_{n}(z)$ be a polynomial in $z$ of degree $n$. If $\left|\Re\left[\pi_{n}(z)\right]\right| \leqq K, z$ on $C$, then $\left|\pi_{n}^{\prime}(z)\right| \leqq K n / \delta_{0}, z$ on $\bar{C}$, where $\delta_{0}$ is the constant which occurs in Definition 4.1 .

THEOREM 4.3. Under the hypothesis of Theorem 4.2 we have

$$
\left|\pi_{n}\left(z_{1}\right)-\pi_{n}\left(z_{2}\right)\right| \leqq L K n\left|z_{1}-z_{2}\right|, \quad z_{1}, z_{2} \text { on } \bar{C},
$$

where $L$ depends only on $C$.

As a converse to Theorem 4.1 we prove

Theorem 4.4. Let $C$ be a curve both of Type D and of Type G. Let $u(z)$ be defined in $\bar{C}$; suppose there exist harmonic polynomials $p_{n}(z)$ such that

$$
\left|u(z)-p_{n}(z)\right| \leqq L / n^{k+1},
$$

z on $\bar{C}$.

Set $f(z)=u(z)+i v(z)$, where $v(z)$ is conjugate to $u(z)$. Then $f(z) \in Z_{A}^{\mathbf{k}}$ on $C$.

Let $z_{0}$ be a point interior to $C$. Set $\pi_{n}(z)=p_{n}(z)+i q_{n}(z)$, where $q_{n}(z)$ is conjugate to $p_{n}(z), q_{n}\left(z_{0}\right)=v\left(z_{0}\right)$.

Consider first the case $k=0$. From (4.1) we obtain

$$
\left|p_{2^{m+1}}(z)-p_{2^{m}}(z)\right| \leqq 2 L / 2^{m}
$$

Set $g_{m}(z)=\pi_{2^{m+1}}(z)-\pi_{2^{m}}(z)$. By (4.2) and Theorem 4.2 we have 


$$
\left|g_{m}^{\prime}(z)\right| \leqq L_{1}
$$

$z$ on $\bar{C}$.

Suppose $C$ has the equation $z=\tau(s)$, where $s$ denotes arc-length measured along $C$. Then from (4.3) and Theorem 4.3 we obtain

$$
\begin{aligned}
\left|g_{m}[\tau(s+h)]+g_{m}[\tau(s-h)]-2 g_{m}[\tau(s)]\right| & \\
& \leqq \int_{s}^{s+h}\left|g_{m}^{\prime}[\tau(\zeta)]-g_{m}^{\prime}[\tau(\zeta-h)]\right| d \zeta \\
& \leqq L_{2} 2^{m+1} \int_{s}^{s+h}|\tau(\zeta)-\tau(\zeta-h)| d \zeta \leqq L_{3} 2^{m+1} h^{2} .
\end{aligned}
$$

We write by virtue of $(4.1)$

$$
u(z)=p_{2}(z)+\sum_{m=1}^{\mu}\left[p_{2^{m+1}}(z)-p_{2^{m}}(z)\right]+\sum_{m=\mu+1}^{\infty}\left[p_{2^{m+1}}(z)-p_{2^{m}}(z)\right],
$$

where $\mu$ is a positive integer to be determined later. We have $\left|p_{2}(z)\right| \leqq L_{4}, z$ on $\bar{C}$; hence by Theorem 4.3

$\left|\pi_{2}[\tau(s+h)]+\pi_{2}[\tau(s-h)]-2 \pi_{2}[\tau(s)]\right|$

$$
\begin{aligned}
& \leqq\left|\pi_{2}[\tau(s+h)]-\pi_{2}[\tau(s)]\right|+\left|\pi_{2}[\tau(s-h)]-\pi_{2}[\tau(s)]\right| \\
& \leqq L_{5}|\tau(s+h)-\tau(s)|+L_{6}|\tau(s-h)-\tau(s)| \leqq L_{7}|h| .
\end{aligned}
$$

From (4.5), (4.4), and (4.2) respectively we thus obtain

$$
\begin{aligned}
\mid u[\tau(s+h)]+u[\tau(s-h)] & -2 u[\tau(s)] \mid \\
\leqq & L_{7}|h|+L_{3} h^{2} \sum_{m=1}^{\mu} 2^{m+1}+8 L \sum_{m=\mu+1}^{\infty} 2^{-m} \\
\leqq & L_{7}|h|+L_{8} 2^{\mu+1} h^{2}+L_{9} / 2^{\mu+1} .
\end{aligned}
$$

Let $h, 0<h<1 / 2$, be arbitrary but fixed. Choose $\mu$ such that $2^{-\mu-1} \leqq h<2^{-\mu}$; it follows that $u(z) \in Z$ on $C$.

Hence by the remark following Theorem 3.6, $f(z) \in Z_{A}$ on $C$, which completes the proof for the case $k=0$.

Suppose now $k \geqq 1$. Then [Walsh, Sewell, and Elliott 1, Theorem 8.3] inequality (4.1) implies $\left|f^{(k)}(z)-\pi_{n}^{(k)}(z)\right| \leqq M / n, z$ on $\bar{C}$. Theorem 4.4 now follows for $k \geqq 1$ by the case $k=0$ just established.

If in Theorem 4.4 we assume the existence of polynomials in $z$ which approximate to $f(z)$ with a degree $1 / n^{k+1}$, then the conclusion may be established without requiring that $C$ be of Type $\mathrm{G}$ :

THEOREM 4.5. Let $C$ be a curve of Type D. Let $f(z)$ be defined in $\bar{C}$; suppose there exist polynomials $\pi_{n}(z)$ in $z$ such that

$$
\left|f(z)-\pi_{n}(z)\right| \leqq L / n^{k+1}, \quad \quad z \text { on } \bar{C} .
$$


Then $f(z) \in Z_{A}^{k}$ on $C$.

We write

$$
f(z)=\pi_{2}(z)+\sum_{m=1}^{\mu} g_{m}(z)+\sum_{m=\mu+1}^{\infty} g_{m}(z) .
$$

Inequalities (4.4) and (4.5) were established without using the hypothesis that $C$ be of Type G. We have $\left|g_{m}(z)\right| \leqq 2 L / 2^{m}$. The conclusion follows from the analogue of (4.6).

5. Problem $\beta$. Let $C$ consist of a finite number of mutually exterior Jordan curves. Let $w=\phi(z)$, with inverse $z=\psi(w)$, map the complement of $\bar{C}$ conformally (not necessarily uniformly) onto $|w|>1$ so that the points at infinity in the two planes correspond to each other. Denote by $C_{R}$ the locus $|\phi(z)|=R>1$ lying exterior to $C$. In Problem $\beta$ we study polynomial approximation on $\bar{C}$ to functions which belong to classes $Z^{k}, Z_{A}^{k}$, and related classes on $C_{R}, R>1$. We shall use

Definition 5.1. A contour is a point set $C$ consisting of the mutually exterior Jordan curves $C_{1}, C_{2}, \cdots, C_{\lambda}$ having the properties

(i) each $C_{j}$ has a tangent at every point;

(ii) the function

$$
\log \left|\frac{\psi(w)-\psi\left(w^{\prime}\right)}{w-w^{\prime}}\right|
$$

for each possible definition of $\psi(w)$ and $\psi\left(w^{\prime}\right)$ is locally bounded in the two-dimensional sense for $|w|=1$ and $\left|w^{\prime}\right| \geqq 1$ in a neighborhood of each point of continuity of $\psi(w)$;

(iii) the function (5.1) satisfies, on each arc $\gamma_{j}$ of $\gamma:|w|=1$ corresponding to a curve $C_{j}$ of $C$, a Lipschitz condition of order unity in w, uniformly with respect to $w^{\prime}$ on any closed arc of $\left|w^{\prime}\right|=1$ interior to $a \gamma_{k}$.

We establish

Theorem 5.1. Let $C$ and $C_{R}, R>1$, be contours. Let $u(z) \in Z^{k}$ on $C_{R}$. Set $f(z)=u(z)+i v(z)$, where $v(z)$ is conjugate to $u(z)$. Then there exist polynomials $\pi_{n}(z)$ such that

$$
\left|f(z)-\pi_{n}(z)\right| \leqq L / n^{k+1} R^{n}, \quad \text { zon } \bar{C} .
$$

The conclusion of Theorem 5.1 has been established [Walsh, Sewell, and Elliott 1, Theorem 10.1] under the stronger hypothesis that $\partial^{k} u / \partial s^{k}$ satisfies a Lipschitz condition of order unity on $C$. The proof given there is valid for the present theorem if in place of Theorem 8.1 [of Walsh, Sewell, and Elliott 1] we apply Theorem 4.1 of the present paper. §9]:

We shall use also the following definition [Walsh, Sewell, and Elliott 1, 
Definition 5.2. A curve $C$ is said to be of Type $\mathrm{B}$ if $C$ is a rectifiable Jordan curve such that for some $q>0$ the integral $\int_{C}\left[\partial g\left(z_{0}, z\right) / \partial \nu\right]^{-q} d s$ exists, where $g\left(z_{0}, z\right)$ is Green's function for the interior of $C$ with pole at the point $z_{0}$, and $\nu$ is the inner normal.

This requirement on $g\left(z_{0}, z\right)$ is independent of $z_{0}$.

As a converse to Theorem 5.1 we prove $\left({ }^{8}\right)$

THEOREM 5.2. Let $C$ be a curve of Type B; suppose that $u(z)$ is defined on $\bar{C}$. Let harmonic polynomials $p_{n}(z)$ exist such that

$$
\left|u(z)-p_{n}(z)\right| \leqq M / n^{k+2} R^{n}, \quad \text { z on } \bar{C}, R>1 \text {. }
$$

Set $f(z)=u(z)+i v(z)$, where $v(z)$ is conjugate to $u(z)$. Then $f(z) \in Z_{A}^{k}$ on $C_{R}$.

By a method previously used [Walsh, Sewell, and Elliott 1, Theorem 10.3] we obtain

$$
\left|f(z)-\pi_{n}(z)\right| \leqq M_{1} / n^{k+1}, \quad z \text { on } \bar{C}_{R},
$$

where $\pi_{n}(z)=p_{n}(z)+i q_{n}(z), q_{n}(z)$ a suitable conjugate of $p_{n}(z)$. Theorem 5.2 now follows from Theorem 4.4.

By the use of properties of functions due to Hardy and Littlewood, Walsh and Sewell [1] introduce a category of classes defined for all integers $p$, positive, negative, or zero, of functions analytic interior to $\gamma:|w|=1$. The function $f(z)$ is said to belong to the class $L(p, 1)$ on $\gamma(p<0)$ if and only if $f(z)$ is analytic interior to $\gamma$ and

$$
\left|f\left(r e^{i \theta}\right)\right| \leqq M(1-r)^{p+1}, \quad 0<r<1 ;
$$

the function $f(z)$ is said to belong to the class $L^{\prime}(p, 1)$ on $\gamma(p \geqq-1)$ if and only if $f(z)$ is analytic interior to $\gamma$ and $f^{(p+2)}(z) \in L(-2,1)$. Thus by Theorem 2.1 the classes $Z_{A}^{p}$ and $L^{\prime}(p, 1)$ are identical, $p \geqq 0$. If $f(z) \in L^{\prime}(p, 1), p \geqq 0$, then (loc. cit.) $f^{(p+2+k)}(z)$ is of class $L(-2-k, 1)$, and the $k$ th indefinite integral of $f(z)$ is of class $L^{\prime}(p+k, 1)$. That is to say, we have a sequence of classes $\cdots, L(-3,1), L(-2,1), L^{\prime}(-1,1), L^{\prime}(0,1)=Z_{A}, L^{\prime}(1,1)=Z_{A}^{1}, \cdots$; for a function which belongs to any of these classes on $\gamma$, the derivative belongs to the next lower class and the indefinite integral to the next higher class.

These classes are extended to more general curves by conformal mapping. Let $C$ be a Jordan curve and let $z=\chi(w)$ map $|w|<1$ onto the interior of $C$. The function $f(z)$ is said to be of class $L(p, 1), p<0$, or $L^{\prime}(-1,1)$ on $C$ if $f[\chi(w)]$ is of class $L(p, 1)$ or $L^{\prime}(-1,1)$, respectively, on $|w|=1 ; f(z)$ is said to be of class $L^{\prime}(p, 1), p \geqq 0$, on $C$ if $f(z)$ is of class $Z_{A}^{p}$ on $C$. Walsh and Sewell [1] define the class $L^{\prime}(p, 1), p \geqq-1$, only for $C$ an analytic Jordan curve; in

(8) Here and in following similar situations we assume that if $u(z)$ or $f(z)$ is not originally supposed to be defined on the entire point set considered, the supplementary definition is to be made by means of the convergent series of polynomials, which is equivalent to defining $u(z)$ by harmonic continuation and $f(z)$ by analytic continuation. 
this case the equivalence of the definition of $L^{\prime}(p, 1), p \geqq 0$, given there, and the definition given here, is a consequence of Theorem 3.4 ; for $p=-1$ the two definitions are identical.

It is shown (loc. cit.) that if $C$ is an analytic Jordan curve and if $f(z)$ is of class $L(p, 1)$ or $L^{\prime}(p, 1)$ on $C_{R}$ according as $p \leqq-2$ or $p>-2$, then there exist polynomials $\pi_{n}(z)$ which converge to $f(z)$ on $C$ with the degree of approximation $1 / R^{n} n^{p+1}$. Reciprocally, if there exist polynomials $\pi_{n}(z)$ which converge to $f(z)$ on $C$ with the degree of approximation $1 / R^{n} n^{p+2}$, then on $C_{R}$ the function $f(z)$ is of class $L(p, 1)$ or $L^{\prime}(p, 1)$ according as we have $p \leqq-2$ or $p>-2$.

Thus the set of classes $Z_{A}^{k}$ admits of a natural extension to negative $k$; the conclusion of Theorem 5.1 has previously been established [for example Walsh and Sewell 1, \$7] for the extended set of classes if $C$ is a contour consisting of a single Jordan curve, but without relating the classes to a condition of form (1.2). We proceed to indicate the properties expressed in Theorem 5.2 for the extended set of classes $(k<0)$ where $C$ is now no longer analytic. A preliminary theorem is needed:

TheOREM 5.3. Let $C$ be a Jordan curve in the $z$-plane; let $z=\chi(w)$ map the interior of $\gamma:|w|=1$ onto the interior of $C$; suppose $\chi^{\prime}(w)$ is continuous and different from zero on $|w| \leqq 1$. Let a family of mutually disjoint analytic Jordan curves $C(\rho)$ interior to $C$ be defined for all values of $\rho$ in an interval $\rho_{0} \leqq \rho<\rho_{1}$ by an equation of the form $C(\rho):|\Phi(z)|=\rho$, where $\Phi(z)$ is analytic in a deleted neighborhood of $C$ interior to $C$, and is continuous in the closure of that neighborhood with $|\Phi(z)|=\rho_{1}$ on $C$, and where $\Phi^{\prime}(z)$ exists and is continuous and different from zero in the closure of that neighborhood. The function $\Phi(z)$ need not be single-valued provided $|\Phi(z)|$ is single-valued.

Then a necessary and sufficient condition that a function $f(z)$ analytic interior to $C$ satisfy the inequality

$$
|f[\chi(w)]| \leqq L_{0}(1-|w|)^{\sigma},
$$$$
\sigma<0,
$$

is that $f(z)$ satisfy the inequality $\left(\rho_{0} \leqq \rho<\rho_{1}\right)$

$$
|f(z)| \leqq L\left(\rho_{1}-\rho\right)^{\sigma},
$$$$
\text { z on } C(\rho) \text {, }
$$

where $L$ is independent of $\rho$.

The essential content of Theorem 5.3 is merely that a condition of form (5.3) is independent of the particular function $\Phi(z)$ in terms of which the $C(\rho)$ are defined, under suitable continuity conditions on $C$ and $\Phi(z)$.

The function $\Phi[\chi(w)]$ is analytic in a deleted neighborhood of $\gamma$ interior to $\gamma$, and is continuous in the closure of that neighborhood with $|\Phi[\chi(w)]|=\rho_{1}$ on $\gamma$; moreover $d \Phi[\chi(w)] / d w$ exists and is continuous and different from zero in the closure of that neighborhood. Consequently it is sufficient to choose, as we do, $C$ identical with $\gamma$, and the $z$ and $w$-planes identical. 
Assume first (5.2) to be satisfied. Let $\rho$ be given, $\rho_{0} \leqq \rho<\rho_{1}$; let $W$ be the point (or one of the points) on $C(\rho)$ which is farthest from the origin; thus $C(\rho)$ lies in the closed region $|w| \leqq|W|$. The inequality $|f[\chi(w)]|$ $\leqq L_{0}(1-|W|)^{\sigma}$ is valid on the circle $|w|=|W|$, hence is valid on $C(\rho)$. By the existence and continuity of $\Phi^{\prime}(w)$ it follows that $\Phi(w)$ satisfies a Lipschitz condition of order unity in a closed neighborhood of $C$ in $\bar{C}$, so we have for $w$ in that closed neighborhood $|\Phi(w)-\Phi(W)| \leqq L_{1}|w-W|$. We choose now $w=W_{1}$ on the half-line from $w=0$ through $W$ and on $C$, whence for $\rho$ sufficiently near $\rho_{1}$,

$$
\begin{aligned}
\rho_{1}-\rho & =|| \Phi\left(W_{1}\right)|-| \Phi(W)|| \leqq\left|\Phi\left(W_{1}\right)-\Phi(W)\right| \\
& \leqq L_{1}\left|W_{1}-W\right|=L_{1}(1-r),
\end{aligned}
$$

from which (5.3) follows.

Assume now (5.3) to be satisfied; let $r$ be given $(0<r<1)$, let the circle $|w|=r$ lie in the neighborhood of $C$ considered, and let $\rho$ be $\max |\Phi(w)|$ on the circle $|w|=r$; thus in some point $W$ we have $|\Phi(W)|=\rho,|W|=r$. On the circle $|w|=r$ we have $|\Phi(w)| \leqq \rho$, so $|w|=r$ lies in the closed interior of $C(\rho)$, and on $|w|=r$ we have (5.3). The function $\omega=\Phi(w)$ maps a deleted neighborhood of $C$ in a locally one-to-one manner onto a deleted neighborhood of $|\omega|=\rho_{1}$; the map is continuous and conformal in the closed region involved. Thus the image of the deleted neighborhood of $C$ covers at least once a neighborhood of $|\omega|=\rho_{1}$, since $\Phi^{\prime}(w) \neq 0$ on $C$. Let $W_{2}$ be the point on $C:|w|=1$ and on the orthogonal trajectory through $W$ of the curves $C(\rho)$, whence $\arg \left[\Phi\left(W_{2}\right)\right]=\arg [\Phi(W)]$; thus $\left|\Phi\left(W_{2}\right)-\Phi(W)\right|=\rho_{1}-\rho$. Since $\Phi^{\prime}(w)$ is bounded from zero we may write for $r$ sufficiently near unity

$$
\begin{gathered}
\left|W_{2}-W\right| \leqq L_{2}\left|\Phi\left(W_{2}\right)-\Phi(W)\right|, \\
1-r \leqq\left|W_{2}-W\right| \leqq L_{2}\left(\rho_{1}-\rho\right),
\end{gathered}
$$

from which (5.2) follows.

Theorem 5.2 for the classes $Z_{A}^{k}$ is complemented by

TheOREM 5.4. Let $C$ be a curve of Type B; let $u(z)$ be defined in $\bar{C}$. Let there exist harmonic polynomials $p_{n}(z)$ such that

$$
\left|u(z)-p_{n}(z)\right| \leqq M / n^{p+2} R^{n}, \quad z \text { on } \bar{C}, R>1, p<0 .
$$

Set $f(z)=u(z)+i v(z)$, where $v(z)$ is conjugate to $u(z)$. Then $f(z) \in L(p, 1)$ on $C_{R}$ if $p \leqq-2$ and $f(z) \in L^{\prime}(-1,1)$ on $C_{R}$ if $p=-1$.

We obtain from (5.4)

$$
\left|p_{n+1}(z)-p_{n}(z)\right| \leqq M_{0} /(n+1)^{p+2} R^{n^{i}}, \quad z \text { on } C .
$$

Let $z_{0}$ be a point interior to $C$; set $\pi_{n}(z)=p_{n}(z)+i q_{n}(z)$, where $q_{n}(z)$ is conjugate to $p_{n}(z), q_{n}\left(z_{0}\right)=v\left(z_{0}\right)$. From (5.5) we obtain [Walsh, Sewell, and Elliott 
1, Theorem 9.6] for $z$ on $C_{\rho_{0}}$, where $\rho_{0}$ is fixed with $1<\rho_{0}<R$, and hence [Walsh 1, p. 77] for $z$ on $C_{\rho}, \rho_{0}<\rho$,

$$
\left|\pi_{n+1}(z)-\pi_{n}(z)\right| \leqq M_{1} \rho^{n+1} /(n+1)^{p+2} R^{n} ;
$$

here $M_{1}$ depends on $\rho_{0}$ but not on $\rho$. The analyticity interior to $C_{\rho}, \rho<R$, of $f(z)=\pi_{2}(z)+\sum_{n=2}^{\infty}\left[\pi_{n+1}-\pi_{n}\right]$ is immediate.

If $p \leqq-2$, it follows [cf. Sewell 1, p. 167] from (5.6) that $|f(z)|$ $\leqq M_{2}(R-\rho)^{p+1}, z$ on $C_{\rho}$. Hence by Theorem 5.3, $f(z) \in L(p, 1)$ on $C_{R}, p \leqq-2$.

If $p=-1$, we obtain from (5.6) and Theorem 4.2

$$
\left|\pi_{n+1}^{\prime}(z)-\pi_{n}^{\prime}(z)\right| \leqq M_{3}(\rho / R)^{n+1}, \quad z \text { on } C_{\rho} ;
$$

here as in the proof of (5.6) we may choose $M_{3}$ independent of $\rho$. Hence (cf. the proof of Theorem 8.3 [Walsh, Sewell, and Elliott 1])

$$
\left|f^{\prime}(z)\right| \leqq M_{4}+M_{3} \sum_{n=2}^{\infty}(\rho / R)^{n+1} \leqq M_{5} /(R-\rho), \quad z \text { on } C_{\rho} .
$$

It now follows from Theorem 5.3 and the definition of $L^{\prime}(-1,1)$ that $f(z)$ $\in L^{\prime}(-1,1)$ on $C_{R}$. The proof of Theorem 5.4 is complete.

The classes $L^{\prime}(-1,1)$ and $L(p, 1), p \leqq-2$, are extended to a set $C$ consisting of a finite number of mutually exterior Jordan curves precisely as were the classes $Z_{\mathbf{A}}^{\boldsymbol{k}}$.

The methods of proof of Theorems 5.2 and 5.4 [see Walsh 1, p. 77] yield

THEOREM 5.5. Let $C$ consist of a finite number of mutually exterior Jordan curves and let $C_{R}$ be a contour. Let $f(z)$ be defined in $\bar{C}$, and let there exist polynomials $\pi_{n}(z)$ such that

$$
\left|f(z)-\pi_{n}(z)\right| \leqq M / n^{p+2} R^{n}
$$

$z$ on $\bar{C}$.

Then on $C_{R}$ we have $f(z) \in Z_{A}^{p}$ if $p \geqq 0, f(z) \in L^{\prime}(-1,1)$ if $p=-1, f(z) \in L(p, 1)$ if $p \leqq-2$.

For the special case that $C$ is the unit circle, Theorems 5.1 and 5.2 can be proved directly, by various methods. Theorems 5.1 and 5.2 do not explicitly contain results on approximation on the line segment $-1 \leqq z \leqq 1$, but such results are easily established by methods previously devised [Walsh, Sewell, and Elliott 1, Theorems 10.2 and 11.2]. We say that a function $u(z)$ belongs to the class $L_{H}^{\prime}(-1,1)$ or $L_{H}(k, 1)$ for $k<-1$ on an analytic Jordan curve $C$ if $u(z)$ is harmonic interior to $C$ and if the corresponding function $f(z) \equiv u(z)$ $+i v(z)$ analytic interior to $C$ belongs to the corresponding class $L^{\prime}(-1,1)$ or $L(k, 1)$ on $C$.

THEOREM 5.6. Let $u(z) \in Z^{k}$ for $k \geqq 0$ or $L_{H}^{\prime}(-1,1)$ for $k=-1$ or $L_{H}(k, 1)$ for $k<-1$ on the ellipse $C_{R}$ whose foci are $z= \pm 1$ and whose semi-sum of axes is $R(>1)$. Set $f(z)=u(z)+i v(z)$, where $v(z)$ is conjugate to $u(z)$ in $C_{R}$. If $P_{n}(z)$ is 
the unique polynomial in $z$ of degree $n$ which interpolates to $f(z)$ in the $n+1$ roots of the polynomial cos $\left[(n+1) \cos ^{-1} z\right]$, then

$$
\left|f(z)-P_{n}(z)\right| \leqq M / n^{k+1} R^{n}, \quad-1 \leqq z \leqq 1 .
$$

THeOREM 5.7. Let $u(z)$ be defined on the interval $-1 \leqq z \leqq 1$, and let there exist real polynomials $p_{n}(z)$ in $z$ such that $(R>1)$

$$
\left|u(z)-p_{n}(z)\right| \leqq M / n^{k+2} R^{n}, \quad-1 \leqq z \leqq 1 .
$$

Then $u(z)$ can be defined with $u(z) \equiv u(\bar{z})$ so as to be of class $Z^{k}$ for $k \geqq 0$ or $L_{H}^{\prime}(-1,1)$ for $k=-1$, or $L_{H}(k, 1)$ for $k<-1$ on the ellipse $C_{R}$ whose foci are \pm 1 and whose semi-sum of axes is $R$.

In Theorem 5.7 of course the analytic function $f(z)=u(z)+i v(z)$, where $v(z)$ is conjugate to $u(z)$ chosen as above, is of the class corresponding to that of $u(z)$ on $C_{R}$; the function $v(z)$ satisfies the equation $v(z) \equiv-v(\bar{z})$.

On the interval $-1 \leqq z \leqq 1$ we have

$$
p_{n}(z) \equiv p_{n}(x) \equiv \sum_{1}^{n} a_{k} x^{k} \equiv \sum_{1}^{n} a_{k} \Re\left[z^{k}\right] \equiv \Re\left[\sum a_{k} z^{k}\right], \Im\left[\sum a_{k} z^{k}\right] \equiv 0 .
$$

Thus on that interval we may interpret $p_{n}(z)$ either as $p_{n}(x)$, or as the values of the polynomial $\pi_{n}(z) \equiv \sum a_{k} z^{k}$, or as the values of the harmonic polynomial $p_{n}(z) \equiv \Re\left[\sum a_{k} z^{k}\right]$.

\section{BIBLIOGRAPHY}

R. NEVANLINNA

1. Eindeutige Analytische Funktionen, Berlin, 1936.

W. SEIDEL

1. Über die Ränderzuordnung bei konformen Abbildungen, Math. Ann. vol. 104 (1931) pp. 182-243.

W. E. SEWELL

1. Degree of approximation by polynomials in the complex domain, Annals of Mathematics Studies, no. 9, Princeton, 1942.

J. L. WALSH

1. Interpolation and approximation by rational functions in the complex domain, Amer. Math. Soc. Colloquium Publications, vol. 20, New York, 1935.

J. L. WALSH and W. E. SEWELL

1. Degree of polynomial approximation to analytic functions-Problem $\beta$, Trans. Amer. Math. Soc. vol. 49 (1941) pp. 229-257.

J. L. Walsh, W. E. Sewell, and H. M. Elliott

1. On the degree of polynomial approximation to harmonic and analytic functions, Trans. Amer. Math. Soc. vol. 67 (1949) pp. 381-420.

S. WARSCHAWSKI

1. Über einen Satz von O. D. Kellogg, Nachr. Ges. Wiss. Göttingen (1932) pp. 73-86.

A. ZYGMUND

1. Smooth functions, Duke Math. J. vol. 12 (1945) pp. 47-76.

HARVARD UNIVERSITY,

Cambridge, Mass. 